\title{
Exclusive Processes at Colliders
}

\author{
A. Szczurek ${ }^{\text {a }}$ \\ anstitute of Nuclear Physics, PL-31-342 Cracow, Poland, \\ University of Rzeszów, PL-35-959 Rzeszów, Poland
}

A few examples of exclusive processes at high energy are discussed. Several mechanisms are presented. The differential distributions are shown. The possibilities to measure the processes are discussed.

\section{Introduction}

Up to now the investigations at high energies were concentrated on inclusive processes, i.e. processes (measurements) when only one object of many produced simultaneously is recorded. Naively, these processes seem to be difficult but can be well described within the standard parton model. The exclusive processes seems naively easier, but in reality require detailed knowledge of the QCD dynamics, which is not required in the inclusive case.

The exclusive production was studied in detail mostly close to the kinematical thresholds. The Tevatron opened a possibility to study the central (semi)exclusive production of mesons (elementary objects) at high energies. A similar program will be carried out in the future at the LHC. Here I review a few examples of exclusive production I have studied recently with my collaborators (for details see Refs. [1/2]3/4 5/6/7/8]).

For exclusive production of a single object (Higgs or meson), the mechanism of the reaction depends on the quantum numbers of the object and/or its internal structure. For heavy scalar mesons (scalar quarkonia, scalar glueballs) the mechanism of the production, shown in Fig [1, is the same as for the diffractive Higgs boson production extensively discussed in recent years [10]. The dominant mechanism for the exclusive heavy vector meson production is quite different. Here the dominant processes are shown in Fig,2, These processes were discussed by A. Cisek during this conference 9].

When going to lower energies the mechanism

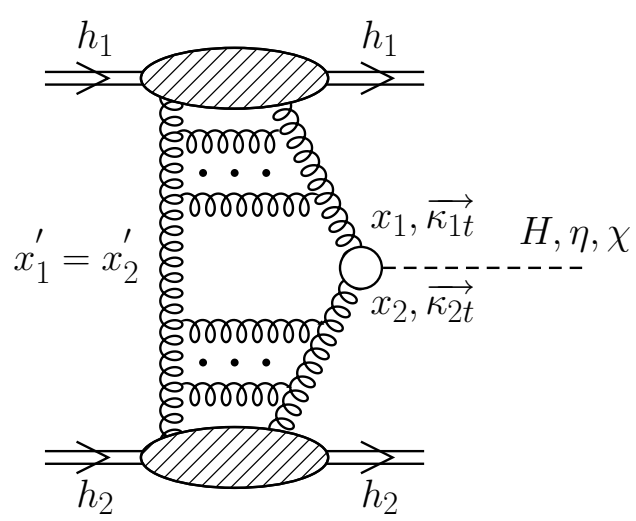

Figure 1. A sketch of the bare QCD mechanism of exclusive heavy scalar meson production.

of the meson production becoming more complicated and the number of mechanisms increases. For example, in Fig 3 I show the mechanism of the glueball candidate $f_{0}(1500)$ production which plays dominant role at low energies [6].

\section{Different examples}

\subsection{Exclusive production of $\chi_{c}$ mesons}

The amplitude of the exclusive double diffractive color singlet production $p p \rightarrow p p \chi_{c J}$ can be written as [10]:

$$
\begin{array}{r}
\mathcal{M}^{g^{*} g^{*}}=\frac{s}{2} \cdot \pi^{2} \frac{1}{2} \frac{\delta_{c_{1} c_{2}}}{N_{c}^{2}-1} \Im \int d^{2} q_{0, t} V_{J}^{c_{1} c_{2}} \\
\frac{f_{g, 1}^{o f f}\left(x_{1}, x_{1}^{\prime}, q_{0, t}^{2}, q_{1, t}^{2}, t_{1}\right) f_{g, 2}^{o f f}\left(x_{2}, x_{2}^{\prime}, q_{0, t}^{2}, q_{2, t}^{2}, t_{2}\right)}{q_{0, t}^{2} q_{1, t}^{2} q_{2, t}^{2}} .
\end{array}
$$



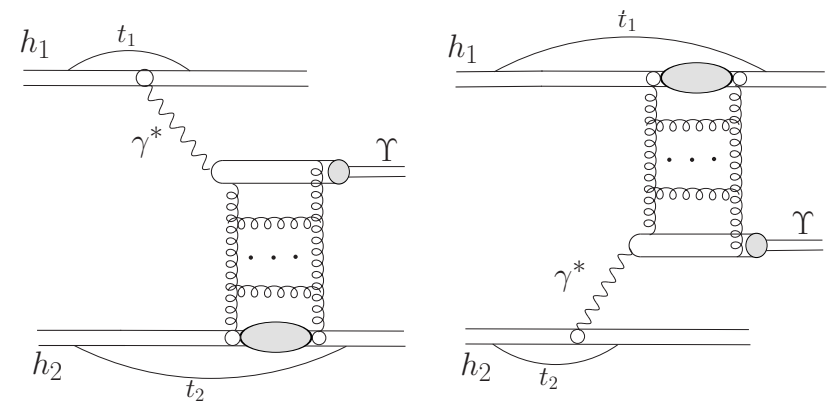

Figure 2. Two basic QED $\otimes$ QCD mechanisms of exclusive heavy vector meson production.

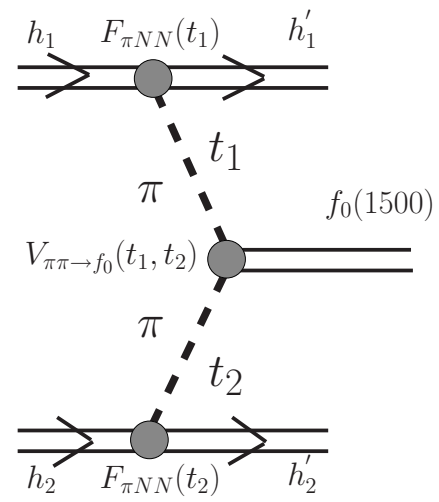

Figure 3. A sketch of the bare QCD mechanism of exclusive heavy scalar $f_{0}(1500)$ meson production.
The amplitude is averaged over the color indices and over the two transverse polarizations of the incoming gluons [10].

In calculating the vertex $V_{J}^{c_{1} c_{2}}$ we have included off-shellness of gluons [3]. The unintegrated gluon distributions were taken from the literature. We have shown in Ref. 3 ] that for relatively light $\chi_{c}(0)$, unlike for the Higgs boson [10, the dominant contributions come from the nonperturbative regions of rather small gluon transverse momenta.

In Ref. 3 we have made a detailed presentation of differential distributions. Here I show only distribution in rapidity of $\chi_{c}\left(0^{+}\right)$(see Fig (4). Although all UGDFs give a similar quality description of the low- $x$ HERA data for the $F_{2}$ structure function, they give quite different rapidity distributions of $\chi_{c}\left(0^{+}\right)$. The UGDFs which take into account saturation effects (GBW, KL) give much lower cross section than the BFKL UGDF (dash-dotted line). Therefore the process considered here would help, at least in principle, to constrain rather poorly known UGDFs.

There is interesting theoretical aspect of the double diffractive production of the $\chi_{c}\left(1^{+}\right)$meson. The coupling $g g \chi_{c}\left(1^{++}\right)$vanishes for onshell gluons (so-called Landau-Yang theorem). According to the original Landau-Yang theorem [11] the symmetries under space rotation and inversion forbid the decay of the spin-1 particle into two (on-shell) spin-1 particles (two photons, two gluons). The same is true for the fusion of two on-shell gluons. The symmetry arguments cannot be strictly applied for off-shell gluons.

In Ref. 4 we have shown explicitly that the Landau-Yang theorem is violated by virtual effects in diffractive production of $\chi_{c}\left(1^{+}\right)$leading to important consequences. In our approach the off-shell effects are treated explicitly. The measurement of the cross section can be therefore a good test of the off-shell effects and consequently UGDFs used in the calculation.

In Fig. 4 I show distributions in rapidity $y$ of $\chi_{c}\left(1^{+}\right)$for different UGDFs from the literature. The results for different UGDFs differ significantly. The biggest cross section is obtained with BFKL UGDF and the smallest cross section with GBW UGDF. The big spread of the results 
is due to quite different distributions of UGDFs in gluon transverse momenta.
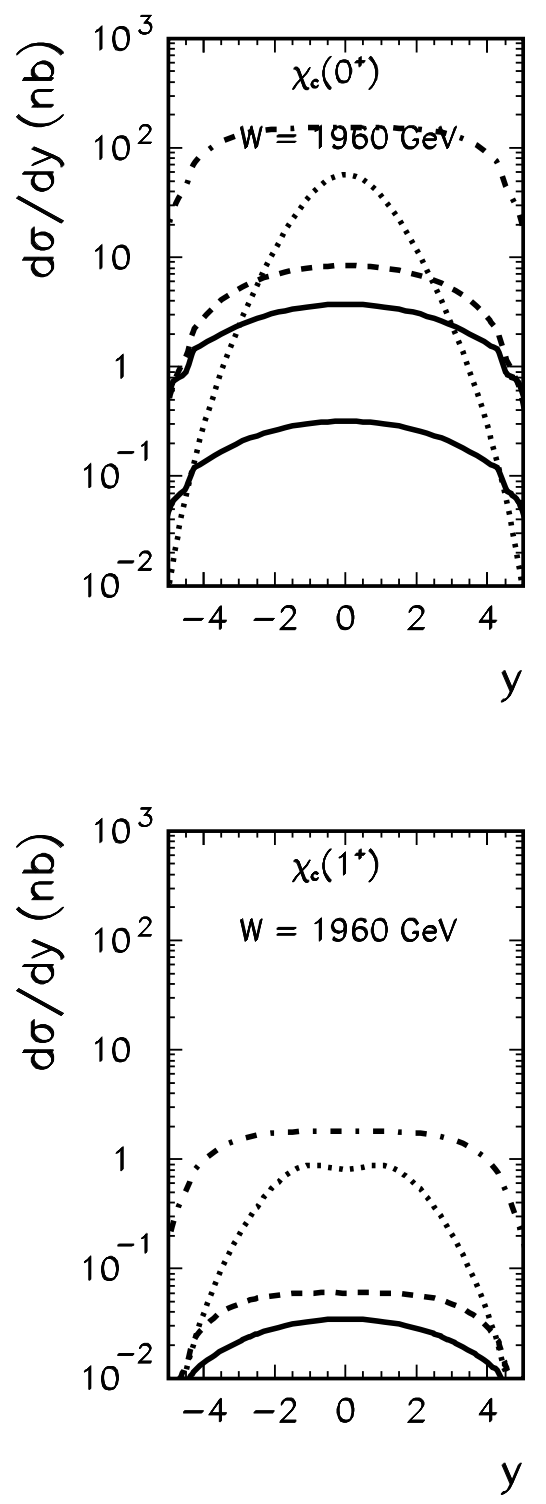

Figure 4. Distribution in rapidity of $\chi_{c}\left(0^{+}\right)$meson (upper panel) and $\chi_{c}\left(1^{+}\right)$meson (lower panel) for different UGDFs.
The cross section for the axial-vector $\chi_{c}\left(1^{+}\right)$ production is much smaller (about two orders of magnitude) than the cross section for the scalar $\chi_{c}\left(0^{+}\right)$production. This smallness can be understood in the context of Landau-Yang theorem, which "causes" vanishing of the cross section for on-shell gluons.

The $\chi_{c}$ mesons are usually measured through the observation of the $\gamma+J / \Psi$ decay channel. The axial-vector $\chi_{c}\left(1^{+}\right)$meson has a large branching fraction for radiative decay $\chi_{c}\left(1^{+}\right) \rightarrow \gamma+J / \psi$ $(\mathrm{BR}=0.36[12])$. This is significantly bigger than for the scalar $\chi_{c}\left(0^{+}\right)$where it is only about $1 \%$ [12. Therefore the discussed off-shell efects are very important to understand the situation in the $\gamma+J / \Psi$ channel observed experimentally. Full analysis requires inclusion of the $\chi_{c}\left(2^{+}\right)$meson where the branching ratio is also relatively high.

\subsection{Exclusive production of the gluball candidate $f_{0}(1500)$}

In Ref. 6] we have discussed exclusive production of scalar $f_{0}(1500)$ in the following reactions:

$$
\begin{aligned}
& p+p \rightarrow p+f_{0}(1500)+p, \\
& p+\bar{p} \rightarrow p+f_{0}(1500)+\bar{p}, \\
& p+\bar{p} \rightarrow n+f_{0}(1500)+\bar{n} .
\end{aligned}
$$

While the first process could be measured at the J-PARC complex being completed recently or by the COMPASS collaboration, the latter two reactions could be measured by the PANDA Collaboration at the new complex FAIR planned in GSI Darmstadt. The combination of these processes could provide more information on the mechanism of $f_{0}(1500)$ production and hopefully some information on its nature.

In Ref. 6] we have proposed a new mechanism (see Fig 3) which becomes dominant at lower energies. In Fig 5 we show the integrated cross section for the exclusive $f_{0}(1500)$ elastic production $p \bar{p} \rightarrow p f_{0}(1500) \bar{p}$ and for double-charge-exchange reaction $p \bar{p} \rightarrow n f_{0}(1500) \bar{n}$. The thick solid line represents the pion-pion component calculated with monopole vertex form factors with $\Lambda=0.8$ $\mathrm{GeV}$ (lower) and $\Lambda=1.2 \mathrm{GeV}$ (upper). The difference between the lower and upper curves represents uncertainties on the pion-pion component. The pion-pion contribution grows quickly 

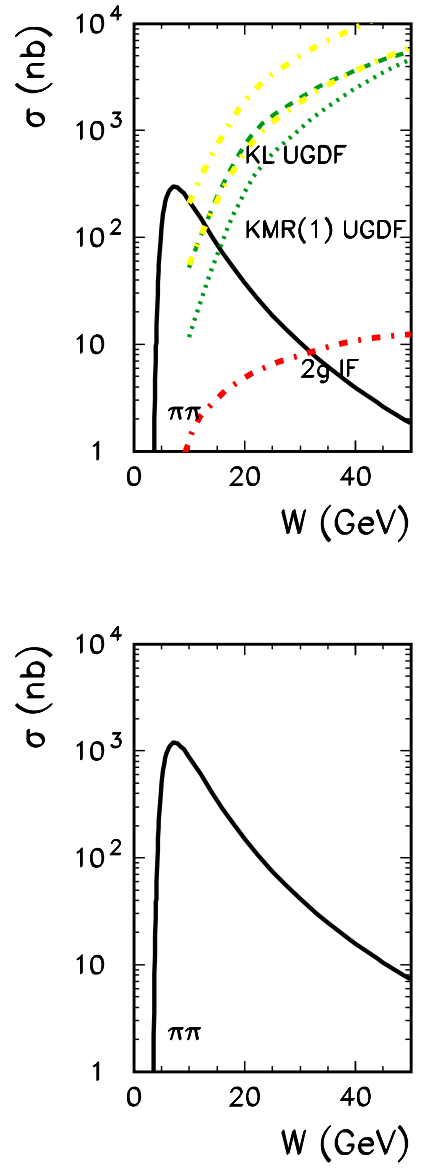

Figure 5. The integrated cross section as a function of the center of mass energy for $p \bar{p} \rightarrow$ $p \bar{p} f_{0}(1500)$ (upper panel) and $p \bar{p} \rightarrow n \bar{n} f_{0}(1500)$ (lower panel) reactions. The thick solid lines are for pion-pion MEC contribution $(\Lambda=0.8$, 1.2 $\mathrm{GeV})$, the dashed line is for QCD diffractive contribution obtained with the Kharzeev-Levin UGDF, the dotted line for the KMR approach and the thin solid lines (blue on-line) are for "mixed" UGDF (KL $\otimes$ Gaussian) with $\sigma_{0}=0.5$, $1 \mathrm{GeV}$. The dash-dotted line represents the twogluon impact factor result [6]. from the threshold, takes maximum at $W \approx 6-7$ $\mathrm{GeV}$ and then slowly drops with increasing energy. The gluonic contribution calculated with unintegrated gluon distributions drops with decreasing energy towards the kinematical threshold and seems to be about order of magnitude smaller than the pion-pion component at $\mathrm{W}=10$ $\mathrm{GeV}$. We show the result with Kharzeev-Levin UGDF (dashed line) which includes gluon saturation effects relevant for small-x, Khoze-MartinRyskin UGDF (dotted line) used for the exclusive production of the Higgs boson and the result with the "mixed prescription" (KL $\otimes$ Gaussian) 6] for different values of the $\sigma_{0}$ parameter: 0.5 $\mathrm{GeV}$ (upper thin solid line), 1.0 GeV (lower thin solid line). In the latter case results strongly depend on the value of the smearing parameter.

\subsection{Exclusive production of the $\pi^{+} \pi^{-}$ pairs}

Up to now I have discussed only exclusive production of a single meson. Also the channels with meson pairs seem interesting. In particular, the channel with two charged pions which seems feasible experimentally.

The underlying mechanism was proposed long ago in Ref. 13]. The general situation is sketched in Fig 6. The corresponding amplitude for the $p p \rightarrow p p \pi^{+} \pi^{-}$process (with four-momenta $p_{a}+$ $\left.p_{b} \rightarrow p_{1}+p_{2}+p_{3}+p_{4}\right)$ can be written as

$$
\begin{aligned}
& \mathcal{M}^{p p \rightarrow p p \pi \pi} \\
& =M_{13}\left(s_{13}, t_{1}\right) F\left(t_{a}\right) \frac{1}{t_{a}-m_{\pi}^{2}} F\left(t_{a}\right) M_{24}\left(s_{24}, t_{2}\right) \\
& +M_{14}\left(s_{14}, t_{1}\right) F\left(t_{b}\right) \frac{1}{t_{b}-m_{\pi}^{2}} F\left(t_{b}\right) M_{23}\left(s_{13}, t_{2}\right),
\end{aligned}
$$

where $M_{i k}$ denotes "interaction" between nucleon $i=1$ (forward nucleon) or $i=2$ (backward nucleon) and one of the two pions $k=\pi^{+}(3), \pi^{-}$(4). In the Regge phenomenology they can be written as a sums of two components:

$$
M_{i k}\left(s_{i k}, t_{1 / 2}\right)=M_{i k}^{R}\left(s_{i k}, t_{1 / 2}\right)+M_{i k}^{P}\left(s_{i k}, t_{1 / 2}\right) .
$$

The first terms describe the subleading reggeon exchanges while the second terms describe exchange of the leading (pomeron) trajectory. The strength parameters of the $\pi N$ interaction are 

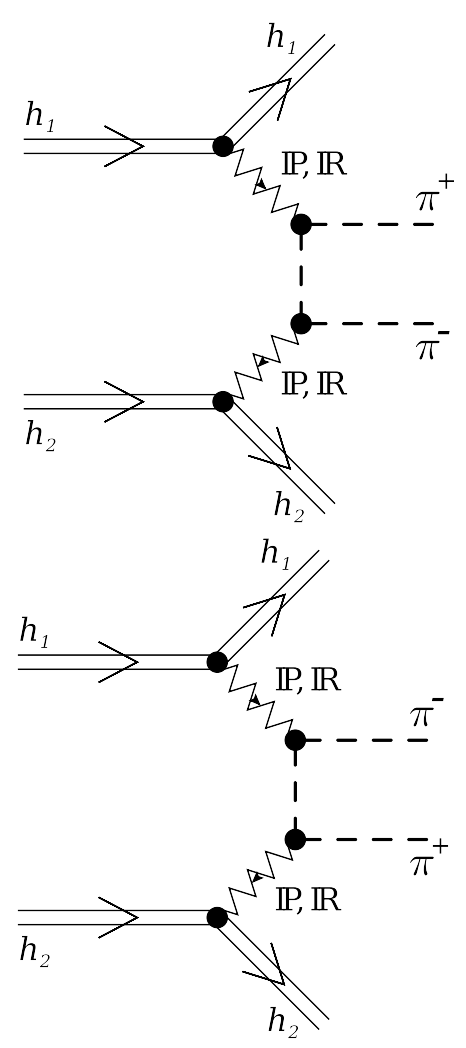

Figure 6. A sketch of the dominant mechanisms of exclusive production of the $\pi^{+} \pi^{-}$pairs at high energies. taken from Ref. 14. We choose post representation of the phenomenological exchange interaction, i.e. interaction for energy in the corresponding final state subsystem. Above $s_{i k}=W_{i k}^{2}$, where $W_{i k}$ is the center-of-mass energy in the $(\mathrm{i}, \mathrm{k})$ subsystem. More details of the calculation will be presented elsewhere [7]. The $2 \rightarrow 4$ amplitude (5) is used to calculate the corresponding cross section including limitations of the four-body phasespace.

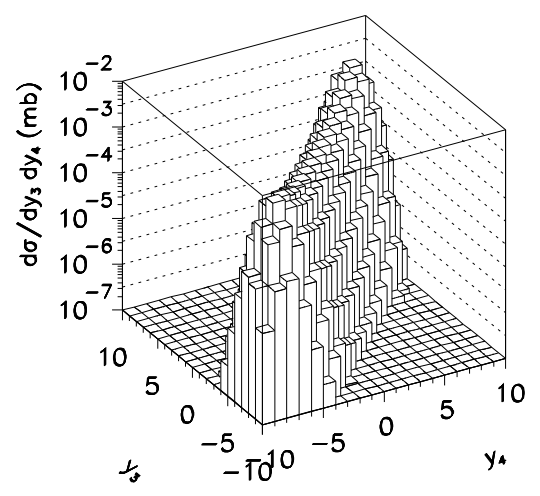

Figure 7. Rapidity distribution of $\pi^{+}$versus $\pi^{-}$for $\mathrm{W}=14 \mathrm{TeV}$.

Here I show only one example of the twodimensional distribution in rapidity of positively charged pion and rapidity of negatively charged pion at the LHC energy of $W=14 \mathrm{TeV}$. The distribution differs considerably from the uniform population of the phase space. One can see a two-dimensional shape of the ridge form elongated along the line $y_{3}=y_{4}$. The minimum of the cross section on the top of the ridge occurs when $y_{3}=y_{4}=0$ and two maxima close to the phase space ends. The minimum occurs in the part of the phase space where the pomeronpomeron contribution dominates, i.e. when both $W_{i k}$ are comparable and large. The maxima are related to the dominance of the pomeron-reggeon and reggeon-pomeron mechanisms, i.e. where one 
of $W_{i k}$ is small and the second one is large. The reggeon-reggeon contribution is completely negligible which is due to the fact that both $W_{i k}$ cannot be small simultaneously. The ALICE collaboration at the LHC should be able to measure such distributions.

\subsection{Exclusive $A A \rightarrow A A \rho^{0} \rho^{0}$}

Exclusive production of elementary particles (lepton pairs, Higgs, etc.) or mesons (vector mesons, pair of pseudoscalar mesons, etc.) in ultrarelativistic heavy-ion collisions is an interesting and quickly growing field [1516/17] of theoretical investigation. On experimental side the situation is slightly different. So far only single- $\rho^{0}$ exclusive cross section $A A \rightarrow A A \rho^{0}$ was measured [18.

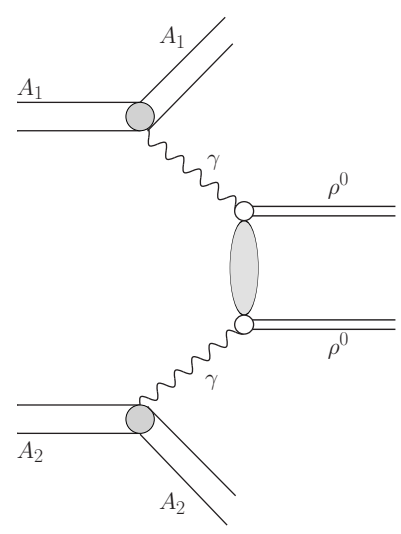

Figure 8. The reaction discussed in this paper.

Let us consider the process $A A \rightarrow A A \rho^{0} \rho^{0}$ depicted in Fig 8 . The cross section takes the familiar form of a convolution of equivalent photon fluxes and $\gamma \gamma$-cross sections. Often flux factors of equivalent, almost on-shell, photons are calculated as for point-like particles with rescaled charge $e \rightarrow Z e$, and the total cross section is calulated using a simple parton-model type formula:

$$
\sigma\left(A A \rightarrow A\left(\rho^{0} \rho^{0}\right) A\right)=
$$

$\int d \omega_{1} d \omega_{2} \frac{n\left(\omega_{1}\right)}{\omega_{1}} \frac{n\left(\omega_{2}\right)}{\omega_{2}} \hat{\sigma}\left(\gamma \gamma \rightarrow \rho^{0} \rho^{0}\right)$.

The formulae (6) clearly does not take into account absorption effects when initial nuclei undergo nuclear breakup. This can be done in the impact parameter space where the geometry of the collision is explicit. Then rather twodimensional flux factors [19] must be used.

The simple EPA formula can be generalized to

$$
\begin{aligned}
& \sigma\left(A A \rightarrow A\left(\rho^{0} \rho^{0}\right) A\right)= \\
& \int d^{2} b_{1} d \omega_{1} d^{2} b_{2} d \omega_{2} N\left(\omega_{1}, b_{1}\right) N\left(\omega_{2}, b_{2}\right) \\
& \theta\left(\left|\vec{b}_{1}-\vec{b}_{2}\right|-R_{12}\right) \hat{\sigma}\left(\gamma \gamma \rightarrow \rho^{0} \rho^{0}\right) .
\end{aligned}
$$

Here the extra $\theta$ function excludes those cases when nuclear collisions, leading to nuclear disintegration, take place $\left(R_{12}=R_{1}+R_{2}\right)$. The twodimensional fluxes in (8) are calculated in terms of the charge form factor of nucleus [20] as:

$N(w, b)=\frac{Z^{2} \alpha}{\pi^{2}} \Phi(x, b)$,

where the auxiliary function $\Phi$ reads:

$\Phi(x, b)=\left|\int_{0}^{\infty} d u u^{2} J_{1}(u) \frac{F\left(-\left(x^{2}+u^{2}\right) / b^{2}\right)}{x^{2}+u^{2}}\right|^{2}$.

The second ingredient of our approach is the $\gamma \gamma \rightarrow \rho^{0} \rho^{0}$ cross section. Here the situation is not well established. The cross section for this process was measured up to $W_{\gamma \gamma}=4 \mathrm{GeV}$ [21. At low energy one observes a huge increase of the cross section.

In Fig 9 we have collected the world data (see 21 and references therein). We use rather directly experimental data in order to evaluate the cross section in nucleus-nucleus collisions. Fig 9 shows our fit to the world data.

The cross section above $W=4 \mathrm{GeV}$ was never measured in the past. It is well known that the cross section for $\gamma \gamma \rightarrow$ hadrons can be well described in the VDM-Regge type model. We use a similar approach for the final state channel $\rho^{0} \rho^{0}$. In Fig 9 I present the corresponding $t$-integrated cross section together with existing experimental data taken from 21]. The vanishing of the VDM-Regge cross section at $W_{\gamma \gamma}=2 m_{\rho}$ 


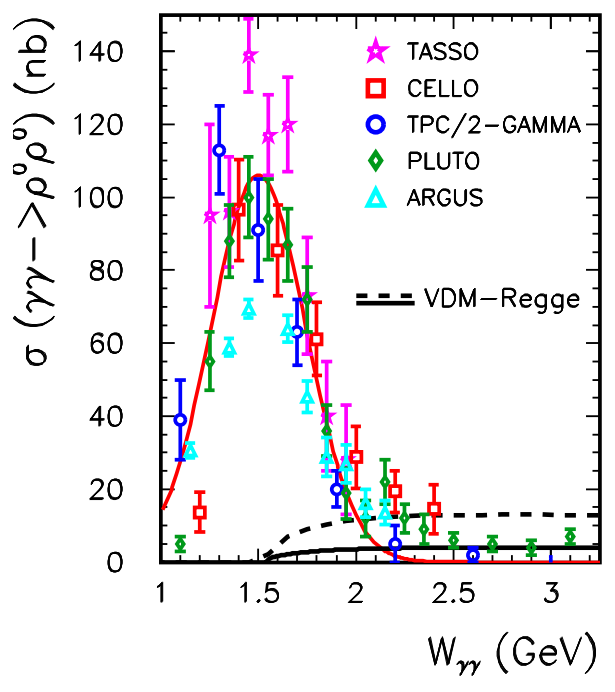

Figure 9. The elementary cross section for the $\gamma \gamma \rightarrow$ $\rho^{0} \rho^{0}$ reaction. We display the collection of the $e^{+} e^{-}$ experimental data 21 and our fit. We show also our predictions based on the VDM-Regge model decribed in [8]. For comparison we show also result when the form factor correcting for off-shell effect is ignored (see [8]).

is due to $t_{\min }, t_{\max }$ limitations. It is obvious from Fig 9 that the VDM-Regge model cannot explain the huge close-to-threshold enhancement. In Fig 10 we show distribution of the cross section for the nucleus-nucleus scattering in photonphoton center-of-mass energy for both low-energy component and high-energy VDM-Regge component. Below $W=2 \mathrm{GeV}$ the low-energy component dominates. The situation reverses above $W$ $=2 \mathrm{GeV}$. One could study the high-energy component by imposing an extra cut on $M_{\rho \rho}$. However, the cross section drops quickly with increasing invariant mass of the $\rho \rho$ pair. The point-like in this (and following) figure(s) means the calculation which excludes regions: $b_{1}<R_{A u}$ and $b_{2}<R_{A u}$.

For illustration in Fig[11 I show the model distribution in impact parameter $b=\left|\vec{b}_{1}-\vec{b}_{2}\right|$. Both

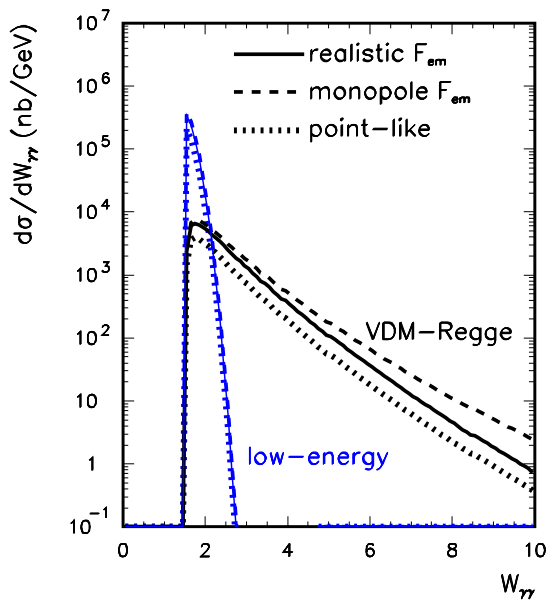

Figure 10. The $A u+A u \rightarrow A u+A u+\rho^{0} \rho^{0}$ cross section as a function of $W_{\gamma \gamma}=M_{\rho \rho}$ for the RHIC energy $\sqrt{s}_{N N}=200 \mathrm{GeV}$. The low- and high-energy components are shown separately.

distributions for the low- and high-energy components are shown separately. I also show distributions for "point-like" charge, monopole form factor and realistic charge density (see [8]). One can see slightly different results for different approaches how to calculate flux factors of equivalent photons.

Finally in Fig 12 I show distribution in rapidity of the $\rho^{0} \rho^{0}$ pair. Compared to the monopole form factor (usually used in the literature), the distribution obtained with realistic charge density is concentrated at midrapidities, and configurations when both $\rho^{0}$ 's are in very forward or both $\rho^{0}$ 's are in very backward directions are strongly damped. A similar effect can be expected for the $A A \rightarrow A A \mu^{+} \mu^{-}$reaction and could be studied by the CMS and ALICE collaborations.

Acknowledgments The results presented here were obtained in collaboration with Wolfgang Schäfer, Roman Pasechnik, Oleg Teryaev, Mariola Kłusek and Piotr Lebiedowicz. This work was partially supported by the Polish Ministry of Science and Higher Education under grants no. N N202 249235 and N N202 078735. 


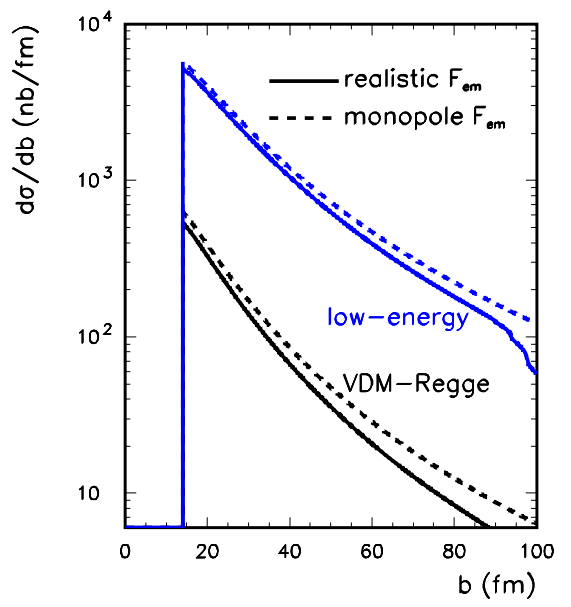

Figure 11. The $A u+A u \rightarrow A u+A u+\rho^{0} \rho^{0}$ cross section as a function of the impact parameter $b$ for $\sqrt{s}_{N N}=200 \mathrm{GeV}$. The meaning of the curves is the same as in Fig 10 The cut off for $R_{12} \approx 14 \mathrm{fm}$ is clearly visible.

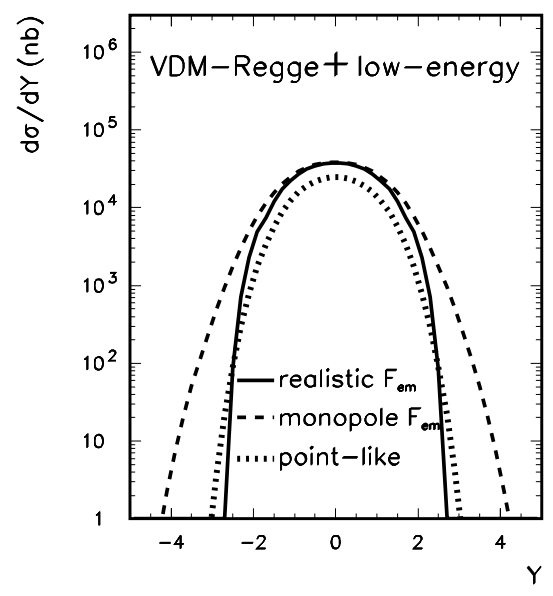

Figure 12. The $A u+A u \rightarrow A u+A u+\rho^{0} \rho^{0}$ cross section as a function of the rapidity of the $\rho^{0} \rho^{0}$ pair $Y$ for $\sqrt{s}_{N N}=200 \mathrm{GeV}$. The meaning of the curves is the same as in Fig 10

\section{REFERENCES}

1. A. Szczurek, R. Pasechnik and O. Teryaev, hep-ph/0608302, Phys. Rev. D75, 054021
(2007).

2. W. Schäfer and A. Szczurek, arXiv:0705.2887, Phys. Rev. D76, 094014 (2007).

3. R. Pasechnik, A. Szczurek and O. Teryaev, Phys. Rev. D78 014007 (2008).

4. R. Pasechnik, A. Szczurek and O. Teryaev, Phys. Lett. B680 6271 (2009). arXiv:0901.4187.

5. A. Rybarska, W. Schäfer and A. Szczurek, arXiv:0805.0717, Phys. Lett. B668 126 (2008).

6. A. Szczurek and P. Lebiedowicz, Nucl. Phys. A826 101 (2009). arXiv:0806.4896.

7. P. Lebiedowicz and A. Szczurek, in preparation.

8. M. Kłusek, W. Schäfer and A. Szczurek, Phys. Lett. B674 92 (2009).

9. A. Cisek, these proceedings.

10. V.A. Khoze, A.D. Martin and M.G. Ryskin, Phys. Lett. B 401, 330 (1997);

V.A. Khoze, A.D. Martin and M.G. Ryskin, Eur. Phys. J. C 23, 311 (2002).

11. L.D. Landau, Dokl. Akad. Nauk. USSR 60 207 (1948);

C.N. Yang, Phys. Rev. 17242 (1950).

12. W. M. Yao et al. (Particle Data Group), Jour. Phys. G33 1 (2006),

C. Amsler et al. (Particle Data Group), Phys. Lett. B667 1 (2008).

13. J. Pumplin and F.S. Henyey, Nucl. Phys. B117 377 (1976).

14. A. Donnachie and P.V. Landshoff, Phys. Lett. B296 227 (1992).

15. V.M. Budnev, I.F. Ginzburg, G.V. Meledin and V.G. Serbo, Phys. Rep. 15181 (1975).

16. G. Baur, K. Hencken, D. Trautmann, S. Sadovsky and Y. Kharlov, Phys. Rep. 364 359 (2002).

17. K. Hencken et al., Phys. Rep. 4581 (2008).

18. B. Grube et al. (STAR collaboration), arXiv:0808.3991 [nucl-ex].

19. J.D. Jackson, Classical Electrodynamics, 2nd ed. (Wiley, New York, 1975), p. 722.

20. G. Baur and L.G. Ferreiro Filho, Phys. Lett. B254 30 (1991).

21. D. Morgan, M.R. Pennington and M.R. Whalley, J. Phys. G20 Suppl. 8A (1994) A1A147. 


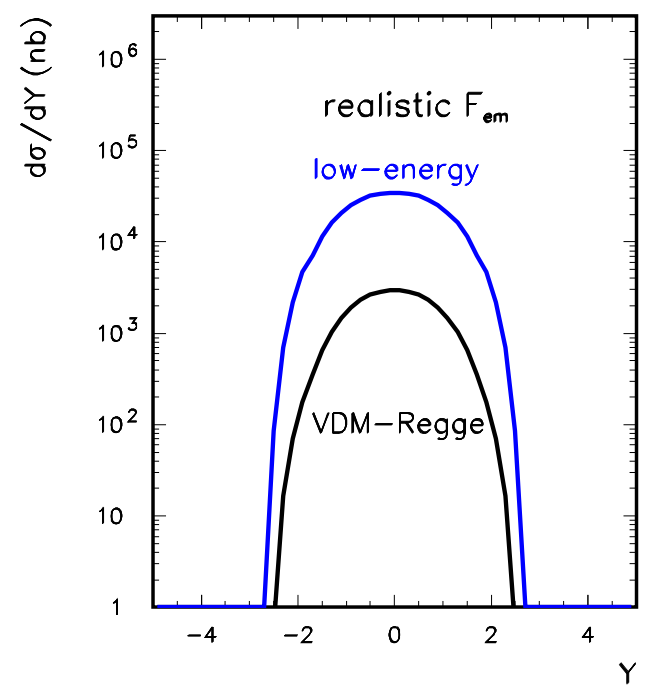

